\title{
Influence of a Changing Gate Bias on the Sensing Properties of SiC Field Effect Gas Sensors
}

\author{
Christian Bur $^{1,2}$, Manuel Bastuck ${ }^{1}$, Mike Andersson ${ }^{2}$, Andreas Schütze $^{1}$, Anita Lloyd Spetz ${ }^{2}$ \\ ${ }^{1}$ Laboratory for Measurement Technology, Saarland University, Campus A5 1, D-66123 Saarbrücken, \\ Germany, \\ c.bur@LMT.uni-saarland.de \\ ${ }^{2}$ Department of Physics, Chemistry, and Biology, Linköping University, SE-581 83 Linköping, Sweden
}

\begin{abstract}
Field effect transistors based on silicon carbide have previously been used with temperature cycled operation to enhance the selectivity. In this study the influence of a changing gate bias on the sensing properties of a platinum gate FET has been studied in order to extend the virtual multi-sensor approach. The sensor exhibits gas specific hysteresis when changing the gate bias indicating that additional information regarding selectivity is contained in the transient behavior. Measurements also showed that especially the shape of the sensor signal changes dramatically with different gas exposures (e.g. $\mathrm{H}_{2}$, CO or $\mathrm{NH}_{3}$ ) during relaxation after step changes of the gate bias. The changing shape primarily reflects the gas itself and not the concentration so that the selectivity of the sensor is increased.
\end{abstract}

Key words: Changing gate bias, MISiC FET, gas specific hysteresis, transient behavior

\section{Introduction}

Field effect devices like MOSFETs can be made gas sensitive by using catalytically-active materials such as palladium, platinum or iridium as metal electrodes. In 1975 Lundström et al. showed [1] that a simple MOSFET with a dense layer of palladium as gate metallization is sensitive towards hydrogen, for a review see [2].

Besides silicon, which was initially used as a substrate material, silicon carbide or other wide band gap materials have provided possibilities to realize sensors for high temperature applications such as combustion control or monitoring of car exhaust gases [3]. The wide band gaps (e.g. $3.2 \mathrm{eV}$ for $4 \mathrm{H}-\mathrm{SiC}$ ) permit their use at elevated temperatures (e.g. up to $800^{\circ} \mathrm{C}$ for $\mathrm{SiC}$ ). In addition, silicon carbide is chemically inert making it suitable for use in harsh environments [2], [3].

However, the basic sensing principle is similar for different kinds of field effect based gas sensors and independent of the materials and the sensor architecture. In respect of a dense, homogeneous, catalytically-active material (commonly $\mathrm{Pt}, \mathrm{Pd}$, or Ir) hydrogen or hydrogen containing gases dissociate on the surface and hydrogen atoms rapidly diffuse through the gate metal creating a polarized layer at the oxide side of the metal/insulator interface [4]. This dipole layer gives rise to an internal potential $V_{G \text {,internal }}$ added to the externally applied gate bias $V_{G}$ (compare Fig. 1a) affecting the density of mobile carriers in the channel region which results in a shift in the IV (current-voltage) curve or CV (capacity-voltage) curve of the device [5]. The sensor signal is then the drain-source voltage $V_{D}$ at a constant drain-current $I_{D}$ or vice versa.

Schalwig et al. [6] suggested that the sensitivity towards hydrogen and non-hydrogen containing gases have a common cause. The mechanism involves spill-over of atomic oxygen from the metal to the oxide surface. The negatively charged oxygen anions will influence the electric field in the semiconductor and thus the IV characteristics of the device. In presence of reducing gases like carbon monoxide (CO), reactions on the catalytic surface will lead to a lower concentration of oxygen and therefore to a change in the sensor signal similar to the mechanism of metal oxide sensors.

The sensitivity and selectivity of the sensor can not only be adjusted by the catalytic material and its morphology [5] but also by the operating parameters like temperature or gate/substrate bias. Nakagomi et al. [7] reported that the threshold voltage and barrier height in the channel change with the gate bias which is also 


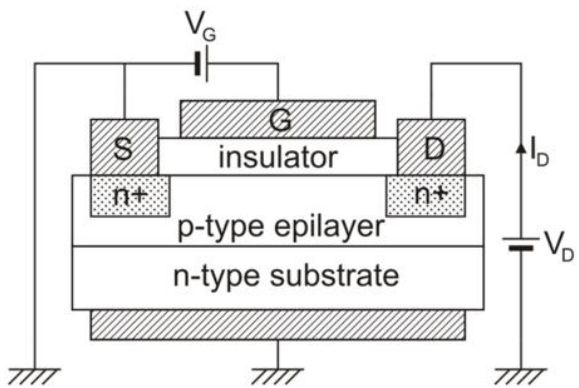

(a)

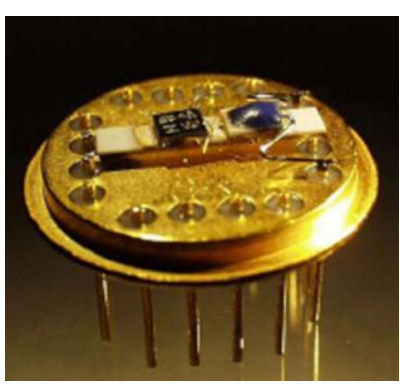

(b)

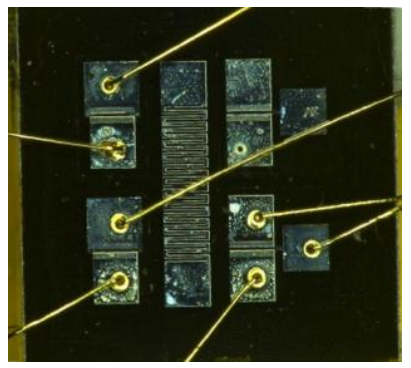

(c)

Fig. 1 Schematic cross-sectional view of the MISiC-FET (a), sensor die with ceramic heater mounted on a TO-8 header (b) and top view of the sensor chip comprising four sensor devices and a Pt-100 temperature sensor (c).

reflected in the gas response. In addition, the temperature has a strong impact on the sensing properties. Thus, in temperature cycled operation (TCO) [8], [9], the sensor is constantly in non-equilibrium and for metal oxide sensors it was reported [10] that there is additional gas significant information contained especially in this transient behavior.

In this paper the influence of a changing gate bias (gate bias cycled operation, GBCO) on the sensing properties is investigated with the aim of further improving the selectivity by extracting additional information from the sensor in a different non-equilibrium state.

\section{Experimental}

For all measurements a SiC based FET (MISiC FET) with porous platinum as catalyticallyactive gate material has been used (Fig. 1). The sensor platform was provided by SenSiC $A B$, Kista, Sweden. The porous gate metallization with a thickness of $25 \mathrm{~nm}$ was deposited at Linköping University, Sweden, in an argon atmosphere at 50 mTorr.

Each sensor die holds a Pt-100 temperature sensor and four transistor devices (Fig. 1c). In two structures the gate and source are connected creating a simple two terminal device (not used in this work). For the other two proper transistors the gate potential $V_{G}$ can be changed individually. The chip with the sensitive layers is glued on a ceramic heater and mounted on a TO-8 header (Fig. 1b). The temperature is controlled by an analogue control circuit using the integrated Pt-100 as a reference.

The sensor was operated at a constant drainsource voltage $V_{D}$ of $4 \mathrm{~V}$, i.e. in the saturation region of the transistor (compare Fig. 2), and the drain-source current $I_{D}$ was measured (compare Fig. 1a). The gate bias $V_{G}$ is changed in the range from $-1 \mathrm{~V}$ to $+5 \mathrm{~V}$ using both continuous ramps as well as step changes at different but constant temperatures in the range of $100^{\circ} \mathrm{C}$ to $300^{\circ} \mathrm{C}$. The obtained measurement data were evaluated by pattern recognition tools (e.g. Linear Discriminant Analysis, LDA) based on shape describing features similar to TCO [9].

\section{Results and Discussion}

Fig. 2 shows the IV-curve of the used sensor device. For an increasing gate bias $V_{G}$ the sensor signal $I_{D}$ increases and for increasing temperatures the signal $I_{D}$ decreases, while the influence of the gate bias becomes weaker for higher temperatures.

The IV curve represents only the static behavior of the sensor in respect of the applied gate bias $\mathrm{V}_{\mathrm{G}}$. Obviously, the gate potential has an impact on the sensing properties. Hence, the transient behavior of the sensor was investigated while $V_{G}$ was continuously changed with increasing and decreasing ramps. Hysteresis of the sensor signal was observed depending on the speed of

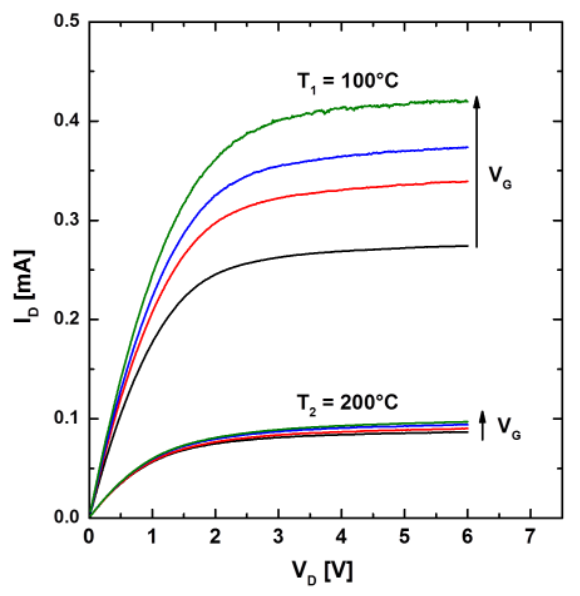

Fig. 2 IV curve showing the influence of changing the operating temperature and external applied gate bias $V_{G}$. 


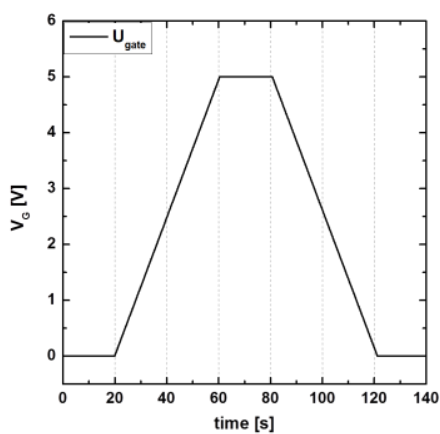

(a)

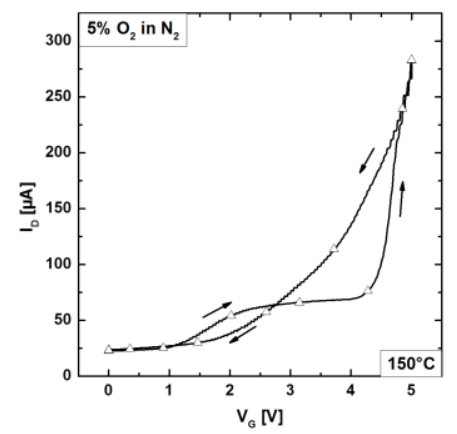

(d)

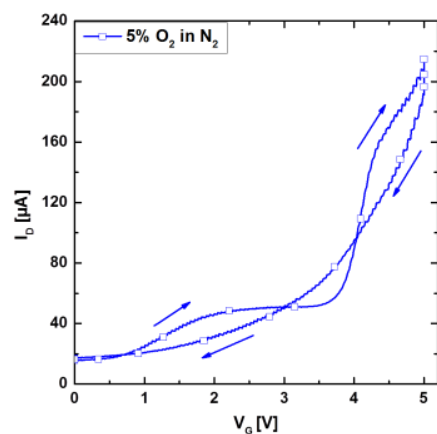

(b)

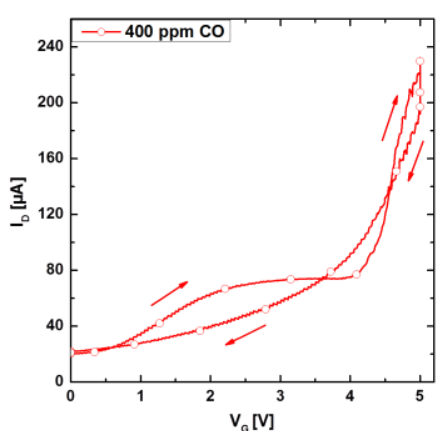

(c)

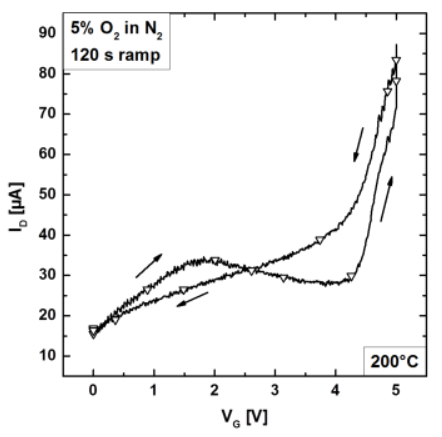

(e)

Fig. 3 Gate bias ramp (40 s) up and down for studying hysteresis (a), hysteresis of the sensor signal in 5\% $\mathrm{O}_{2}$ atmosphere at $200^{\circ} \mathrm{C}$ (b) and hysteresis when $400 \mathrm{ppm} \mathrm{CO}$ is added (c). Hysteresis of the sensor signal at $150^{\circ} \mathrm{C}(\mathrm{d})$ and with a $120 \mathrm{~s}$ ramp (e). Measurements were taken every $100 \mathrm{~ms}$, symbols are indicative only.

the ramps (Fig. 3): it was found that (strong) hysteresis only occurs for high speed ramps (but still in the order of seconds). Furthermore, the hysteresis changes with different gas exposures indicating that the sensor is in nonequilibrium and that this is affected by the adsorbed species. Especially the path corresponding to increasing $V_{G}$ shows a visibly non-monotonous behavior resulting in two crossing points with the path for decreasing $U_{G}$ (Fig. 3, b-e). Gas adsorption on the sensor surface shifts the crossing points to higher values of the gate bias (shown for $\mathrm{CO}$ in Fig. 3c) and thus, changes the hysteresis curve. In addition, the hysteresis depends on the operating temperature (Fig. 3d) as well as on the speed of the ramp. In principle, the hysteresis cannot be explained by the normal behavior of MOSFETs but instead with gas adsorption on the catalytically-active layer. Due to the dependence on temperature and speed of the ramp (which is still in the orders of several seconds) it can be assumed that spillover effects of adsorbed molecules or other slow relaxation processes taking place on the surface are responsible for the observed behavior.

From former studies dealing with temperature hysteresis of metal oxide sensors it is known that transient behavior provides additional information about the gas atmosphere which is maximized for maximum hysteresis [10]. Based on this approach, a simple gate bias cycle with step changes was studied covering a bias range from -1 to $+3 \mathrm{~V}$ at different temperatures (Fig. 4). After a change of the bias voltage the sensor signal exhibits a slow relaxation (on the order of approx. 10 seconds). We observed that different gas exposures have a strong impact on this relaxation. In particular, hydrogen suppresses the peak at the beginning of each voltage plateau while carbon monoxide changes the relaxation time compared to the response in an oxygen-nitrogen atmosphere. The observed behavior when changing the gate bias is consistent with the hysteresis behavior observed for the gate bias ramps.

For a small positive bias the sensor is less gassensitive which is due to the fact that the used device is on the edge of a normally-on device. That means, at zero applied bias there is only a very small conducting channel (small amount of mobile carriers) and the effect of adsorbed gas molecules is not strong enough to affect the channel. Furthermore, a negative bias voltage pinches off the channel completely resulting in nearly zero current. However, a negative applied voltage still influences the interaction of the gas molecules with the catalytic layer as can be seen by the relaxation behavior after changing the bias. 

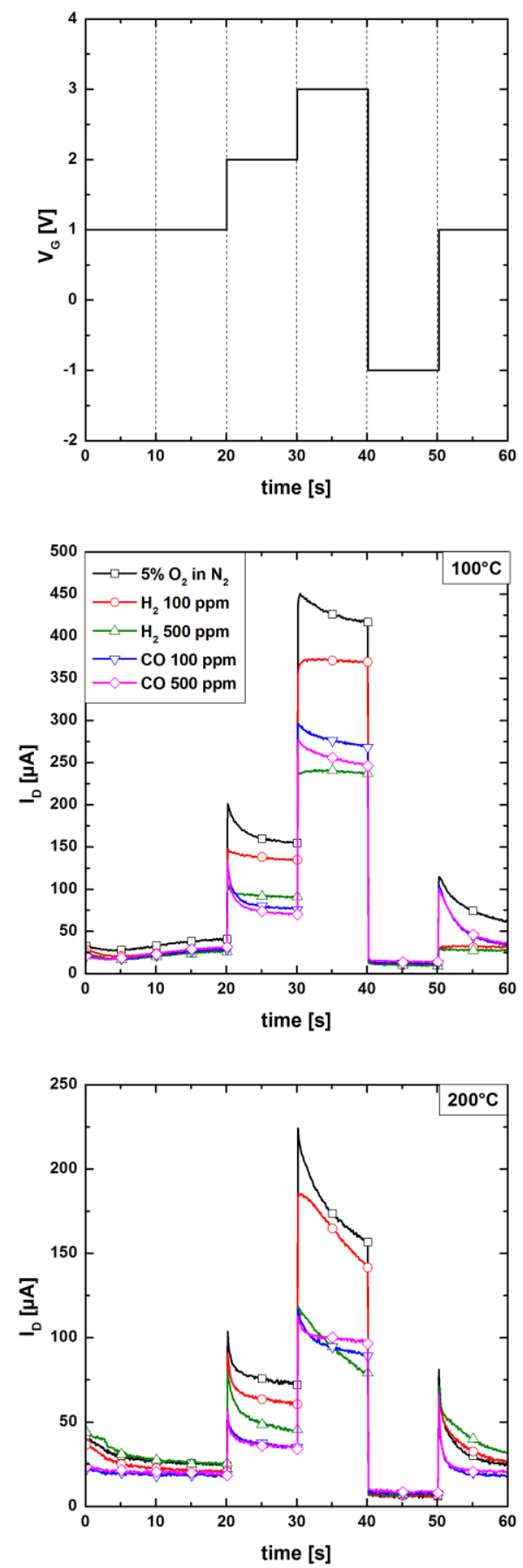

Fig. 4 Gate bias cycle applied to the sensor at different temperatures, resulting sensor signals at $100^{\circ} \mathrm{C}$ and $200^{\circ} \mathrm{C}$ in $5 \% \mathrm{O}_{2}$ and with admixture of $\mathrm{H}_{2}$ and $\mathrm{CO}$.

Exemplary data evaluation was performed with linear discriminant analysis using features describing the shape of the sensor response [7]. For each $V_{G}$ plateau the slope and the mean value of $I_{D}$ were calculated and used for discrimination. As shown in Fig. 5 the features allow both discrimination of the tested gases as well as separation of the different concentrations.

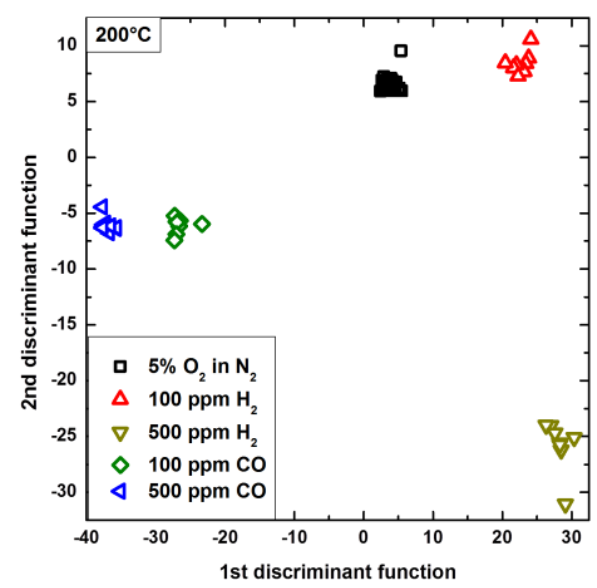

Fig. 5 LDA discriminating hydrogen and carbon monoxide in a dry atmosphere of $5 \%$ oxygen.

\section{Conclusion and Outlook}

The influence of a changing gate bias on the sensing properties of a Pt-gate field effect sensor has been investigated. The results suggest that additional information is contained in the transient response of the sensor allowing an increase of the selectivity. In fact, GBCO can be used in a similar way to TCO. Future work will address a combination of TCO and GBCO.

\section{Acknowledgements}

The authors would like to thank SenSiC $A B$, Kista, Sweden, for providing the sensors and $3 S$ - Sensors, Signal Processing, Systems $\mathrm{GmbH}$, Saarbrücken, Germany for providing the hardware for sensor control and data read-out.

\section{References}

[1] I. Lundström et al., Appl. Phys. Lett. 26, 55 (1975), doi: 10.1063/1.88053

[2] I. Lundström et al. Sens. and Actuators B 121 (2007), doi: 10.1016/j.snb.2006.09.046

[3] A. Lloyd Spetz, S. Savage in Recent Major Advances in SiC (2003), ISBN: 3-540-40458-9

[4] M. Eriksson et al., J Appl. Phys. 83, 3947-3951 (1998), doi: 10.1063/1.367150

[5] Z. Darmastuti et at., Proc. IEEE Sensors (2011), doi: 10.1109/ICSENS.2011.6127261

[6] J. Schalwig et al., IEEE Sens. J. 2, 5 (2002), doi: 10.1021/ac60214a047

[7] S. Nakagomi et al., Sens. and Actuators B 108 (2005), doi: 10.1016/j.snb.2004.11.057

[8] C. Bur et al., IEEE Sens. J., in press, doi: 10.1109/JSEN.2011.2179645

[9] C. Bur et al., Microsystem Technologies (2011), doi: 10.1007/s00542-012-1434-z

[10] A. Schütze et al., IEEE Sens. J. 4, 6 (2004), doi: 10.1109/JSEN.2004.833514 